\title{
EFECTOS DE UNA INTERVENCIÓN COGNITIVO CONDUCTUAL Y ACTIVIDAD FÍSICA SOBRE VARIABLES MÉDICAS Y PSICOLÓGICAS EN PACIENTES SOMETIDOS A TRASPLANTE HEMATOPOYÉTICO DE CÉLULAS MADRE
}

\author{
EFFECTS OF A COGNITIVE-BEHAVIORAL INTERVENTION AND PHYSICAL ACTIVITY \\ ON MEDICAL AND PSYCHOLOGICAL VARIABLES IN PATIENTS TREATED WITH \\ HEMATOPOIETIC STEM CELL TRANSPLANTATION
}

\author{
Liliana Mey Len Rivera-Fong', Edgar Landa Ramírez', Luis Manuel Valero Saldaña ${ }^{2}$ y \\ Angélica Riveros Rosas ${ }^{1}$ \\ ' Facultad de Psicología, Universidad Autónoma de México. México. \\ 2 Unidad de Trasplante de Médula Ósea. Instituto de Cancerología de México. México.
}

Resumen

Objetivo: Evaluar el efecto de una intervención cognitivo conductual y un programa de actividad física sobre variables psicológicas y médicas en pacientes sometidos a trasplante de células progenitoras hematopoyéticas (TCPH). Método: Diseño $\mathrm{N}=1$ con grupo control histórico, participaron 14 adultos con quienes se implementó psicoeduación y relajación progresiva muscular pasiva previo a la hospitalización y se realizó un programa de actividad física durante la estancia hospitalaria. Se midieron variables psicológicas mediante la Escala Hospitalaria de Ansiedad y Depresión (HADS) al momento de la evaluación, día de hospitalización, día de alta y primer consulta de seguimiento y con el Termómetro de Estrés (DT) en la evaluación y todos los días durante la hospitalización. Por otro lado se creó un grupo control histórico con 21 pacientes tratados en la misma unidad, con quienes se compararon el número de interconsultas a psiquiatría, el requerimiento farmacológico y los días de estancia hospitalaria.

Resultados: En los sujetos que se beneficiaron de la intervención, se observó que ansiedad, depresión y estrés, tuvieron variaciones que se mantuvieron en rangos normales. Al comparar entre grupos se observó que en el
Abstract

Purpose: The purpose of the study was to evaluate the effect of a cognitive behavioral intervention and a physical activity program on psychological and medical variables in patients undergoing hematopoietic stem cell transplantation (HSCT).

Method: The design used was $N=1$ with historical control group, participants were 14 adults. The intervention consisted in psicoeducation and passive progressive muscle relaxation training prior to hospitalization, and a program of physical activity during the hospital stay. Psychological variables were measured trough the Hospital Anxiety and Depression Scale (HADS) in four different moments: baseline assessment, hospital admission, hospital discharge, and first followup consultation. The Distress Thermometer (DT) was also used in the baseline assessment and every day during hospitalization. On the other hand, the historical control group consisted of 21 patients treated in the same unit. We compared: number of interconsultations to psychiatry, drug requirement and number of days in hospitalization.

Results: The differences observed were: in the intervention group, the number of patients requiring a psychiatric consultation

Correspondencia:

Liliana Mey len Rivera-Fong

Sebastián Lerdo de Tejada No. 51 Edif. B3 Acc. C Depto. 201. Colonia Campamento 2 de octubre, Delegación Iztacalco, C.P. 08930. México. E-mail: liliana_medicinaconductual@hotmail.com 
grupo de intervención fue menor el número de pacientes que requirieron interconsultas a psiquiatría, menor número de interconsultas y una estancia hospitalaria más corta en comparación con grupo control.

Conclusiones: La intervención permitió mantener la estabilidad psicológica de los pacientes, así como menor comorbilidad psiquiátrica y farmacológica que facilita el seguimiento y al reducir la estancia hospitalaria se reducen costos y alteraciones psicosociales en el paciente.

Palabras clave: Trasplante de médula ósea, terapia cognitivo-conductual. Intervención multicomponente. was lower, the patients who were referred to psychiatry required less interconsultations, and hospital stay was shorter compared with control group.

Conclusions: It was concluded that the intervention allowed maintain psychological stability of patients and minor psychiatric and pharmacological comorbidity facilitates monitoring and reduce hospitalization costs and reduced psychosocial distress in the patient.

Key words: Bone marrow transplantation, cognitive-behavioral therapy, multimodal intervention.

\section{INTRODUCCIÓN}

La Sociedad Americana para el Cáncer define al trasplante de células progenitoras hematopoyéticas (TCPH) como un procedimiento médico utilizado para restaurar las células madre sanguíneas cuando la médula ósea ha sido destruida debido a enfermedad, quimioterapia o radiación ${ }^{(1)}$. Debido a las altas dosis de quimioterapia que se utilizan y su eficacia en la eliminación de células oncológicas ${ }^{(2)}$ este procedimiento ha sido considerado parte importante del tratamiento de algunos tipos de cánceres hematológicos, como linfomas, leucemias y mieloma múltiple; por lo cual, cada vez es más frecuente su realización a nivel internacional(2,3) y nacional(4).

Es un tratamiento que ofrece la probabilidad de cura, pero a su vez se ha encontrado que impacta la calidad de vida de los pacientes y repercute en el desarroIlo de morbilidad psicosocial ${ }^{(2,5)}$ debido a que conlleva posibles efectos secundarios agudos y crónicos como enfermedad injerto contra huésped, infecciones, menopausia prematura, osteoporosis, segundas neoplasias, problemas de funcionamiento pulmonar e infertilidad ${ }^{(2,6,7)}$; así como factores biopsicosociales adversos como aislamiento, deprivación social y altas demandas conductuales primordialmente de higiene y alimentación ${ }^{(8)}$ con el fin de evitar complicaciones agudas como infecciones, mucositis y cistitis hemorrágica que prolongan la estancia hospitalaria y comprometen el éxito del trasplante(2).

En este sentido se ha documentado que al momento de la evaluación pre-trasplante, el $50 \%$ de los candidatos reportan distrés y niveles de bajos a moderados en ansie$\operatorname{dad}^{(9)}$, mientras que durante el periodo de hospitalización, se ha observado la presencia, mantenimiento o exacerbación de sintomatología de ansiedad y depresión, que puede integrar criterios diagnóstico para trastornos mentales, siendo el más frecuente el trastorno adaptativo hasta en el $25 \%$ de los pacientes ${ }^{(5)}$. Por otro lado, la falta de adherencia a tratamiento durante el procedimiento se ha relacionado con complicaciones tanto en el éxito de trasplantes como en el desarrollo de lesiones crónicas ${ }^{(10)}$.

En relación a los mediadores psicológicos durante el proceso de trasplante, se tiene evidencia sólida de que el distrés psicológico pretrasplante predice distrés psicológico postrasplante ${ }^{(11)}$, por otro lado, el redefinir la situación tiene una correlación negativa moderada con el número 
de días requeridos para el injerto y una correlación positiva con los niveles de hemoglobina, lo que acelera la recuperación del paciente ${ }^{(12)}$, mientras que el uso de estrategias de relajación se correlaciona negativamente con el número de infecciones durante el trasplante ${ }^{(12)}$, así como un estilo de afrontamiento evitativo incrementa los síntomas comunes de cáncer en los seis meses siguientes al TCPH ${ }^{(12)}$ y tiene una correlación positiva con el desarrollo de trastorno de estrés postraumático ${ }^{(13)}$. Mientras que el estado funcional físico y emocional del paciente han mostrado una correlación alta con autorreporte de recuperación durante el trasplante ${ }^{(14)}$. Con base en estos datos, se ha hecho énfasis en la necesidad de realizar una evaluación integral de los pacientes y que reciban apoyo psicológico durante el proceso de $\mathrm{TCPH}^{(5,15)}$.

En este sentido la terapia cognitivo conductual (TCC) ha mostrado consistentemente efectos benéficos en depresión, ansiedad, trastornos del dormir, fatiga, dolor y estrés en pacientes oncológicos ${ }^{(16)}$. En el caso específico de TCPH se han realizado diversos estudios de intervención con variantes en el foco de atención; algunos se han centrado en brindar información clara y oportuna al paciente sobre su padecimiento y el $\mathrm{TCPH}^{(17)}$; otras han demostrado que la relajación es eficiente para el manejo de estrés, ansiedad y depresión durante la estancia hospitalaria ${ }^{(18,19)}$. Por otro lado, el ejercicio físico durante el periodo de internamiento, se ha relacionado con mejoría en la calidad de vida del paciente ${ }^{(20)}$, además de tener efectos positivos en fatiga, capacidad física y reducción del estrés ${ }^{(21)}$; mientras que la literatura informa que la inmovilización por reposo en cama en personas sanas produce pérdida de masa y fuerza muscular proporcional al periodo de inmovilización, así como reducción de la función cardiovascular ${ }^{(22)}$.

Finalmente se han implementado programas multicomponentes que incluyen psicoeducación sobre la capacidad física, relajación progresiva muscular y un programa de actividad física los cuales han reportado mejoría significativa en la conservación de la fuerza muscular ${ }^{(23)}$ disminución en la intensidad de la diarrea ${ }^{(24)}$, menor tiempo de requerimiento de nutrición parenteral(24) y menor pérdida de funcionalidad física ${ }^{(23)}$. En relación a la ansiedad y la depresión, no se encontraron diferencias entre el grupo experimental y el grupo control, cabe resaltar que en ambos grupos se mantuvieron en rangos normales durante el proceso de trasplante; sin embargo, se observa una tendencia a favor del grupo de intervención en calidad de vida y funcionamiento psicológico ${ }^{(24)}$.

En los estudios de intervención multicomponentes, un porcentaje amplio de sus participantes realizaban actividad física con regularidad antes del trasplante ${ }^{(23,24)}$ mientras que en la población adulta sana mexicana, la prevalencia de inactividad física aumentó $47,3 \%$ en los últimos seis años $^{(25)}$, en población oncológica no se cuenta con datos. Por otro lado, la evidencia sobre su impacto en variables psicológicas como ansiedad y depresión aún es limitada, ya que sólo Kim y Kim han encontraron diferencias en estas variables ${ }^{(19)}$, mientras que los demás estudios no las consideraron o no encontraron diferencias. Además no se ha tomado en cuenta su relación con variables médicas como estancia hospitalaria y requerimiento psicofarmacológico debido a comorbilidad psiquiátrica.

En virtud de lo anterior el objetivo de este estudio fue evaluar el efecto de una intervención cognitivo conductual y actividad física sobre variables psicológicas (ansiedad, estrés y depresión) y médicas (días de estancia hospitalaria, interconsultas a psiquiatría, medicamentos psiquiátricos indicados durante la hospitalización) en pacientes hospitalizados para TCPH. 


\section{MÉTODO}

Este estudio fue desarrollado en el principal centro de trasplantes hematopoyético de células madre de México, el Instituto Nacional de Cancerología, en donde fue aceptado por los Comités Científicos y de Ética.

Se incluyeron a pacientes adultos que fueron enviados a la Unidad de Trasplante de Médula Ósea (UTMO), durante los meses de septiembre del 2012 a enero de 2013. Basados en las condiciones mínimas requeridas para llevar a cabo el programa de actividad física, se consideraron como criterios de exclusión a los pacientes con algún trastorno motor o metástasis a hueso; así como cualquier contraindicación del médico tratante para realizar actividad física.

Se conformó un grupo histórico integrado por pacientes sometidos a TCPH de marzo a agosto del 2012; se consideraron estos participantes ya que fueron tratados por el mismo equipo de salud (médicos y enfermeras), lo que permitió homogenizar los criterios de atención y toma de decisiones.

Se trató de un diseño $\mathrm{N}=1$ con réplicas con grupo control histórico; en el que se llevó a cabo una intervención cognitivo conductual más un programa de actividad física; se midió ansiedad y depresión en cuatro momentos (evaluación, ingreso a la Unidad de Trasplante de Médula Ósea (UTMO), al alta y en la primer consulta de seguimiento) y estrés en la evaluación inicial y todos los días durante la estancia hospitalaria. Para evaluar el impacto de las variables psicológicas en la condición médica del paciente, se tomaron de los expedientes físico y electrónico de los participantes, tanto del grupo de intervención como del grupo control histórico, las interconsultas a psiquiatría, el manejo psicofarmacológico y los días de estancia hospitalaria.

\section{Intervención cognitivo conductual}

Los pacientes que decidieron no participar en el estudio fueron evaluados por psicólogos del servicio de psicooncología del hospital. En los sujetos que accedieron a participar, tras firmar el consentimiento informado se recabaron sus datos sociodemográficos y médicos y se hizo una eva-

\section{Tabla 1. Intervención cognitivo conductual durante trasplante hematopoyético de células madre}

\begin{tabular}{|c|c|c|c|c|}
\hline Sesión & Técnica & Objetivo & Material & Duración \\
\hline 1 & Psicoeducación & $\begin{array}{l}\text { Fomentar y promover la salud } \\
\text { a través de la modificación } \\
\text { de actitudes, aptitudes y } \\
\text { hábitos, en sentido positivo e } \\
\text { incrementar el cumplimiento } \\
\text { terapéutico. }\end{array}$ & $\begin{array}{l}\text { Manual: "Guía para } \\
\text { pacientes candidatos } \\
\text { a trasplante de médula } \\
\text { ósea" (Diseñada para el } \\
\text { presente estudio) }\end{array}$ & $\begin{array}{l}60-90 \\
\text { minutos }\end{array}$ \\
\hline $2-4$ & $\begin{array}{l}\text { Relajación } \\
\text { progresiva } \\
\text { muscular }\end{array}$ & $\begin{array}{l}\text { Disminuir la sensación } \\
\text { subjetiva de ansiedad } \\
\text { dependiente de la tensión } \\
\text { muscular. }\end{array}$ & $\begin{array}{l}\text { Versión adaptada de la } \\
\text { técnica propuesta por } \\
\text { Schwartz y Haynes en } \\
1974 .\end{array}$ & $\begin{array}{c}20 \\
\text { minutos }\end{array}$ \\
\hline Hospitalización & $\begin{array}{l}\text { Programa de } \\
\text { actividad física }\end{array}$ & $\begin{array}{l}\text { Mantener actividad física } \\
\text { en los sujetos durante la } \\
\text { hospitalización. }\end{array}$ & $\begin{array}{l}\text { Rutina de } 15 \\
\text { ejercicios simples de } \\
\text { estiramiento, todos se } \\
\text { realizan mientras el } \\
\text { paciente está sentado. }\end{array}$ & $\begin{array}{c}20 \\
\text { minutos }\end{array}$ \\
\hline
\end{tabular}


luación de las variables de interés; posterior a ello se implementó una intervención cognitivo conductual descrita en la tabla 1 ; que estuvo conformado por psicoeducación y relajación progresiva muscular pasiva llevadas a cabo en consulta externa, para lo cual se agendaron fechas que coincidieran con citas médicas del paciente con el fin de no incrementar sus visitas al Hospital. Además de un programa de actividad física diseñado para pacientes que no realizan ejercicio con regularidad ${ }^{(26)}$, el cual se inició el día de ingreso a la UTMO y se llevó a cabo durante toda la estancia hospitalaria, a excepción de los días que los resultados de laboratorio reportaron recuento menor a 10.000 plaquetas.

Debido a la gran demanda del servicio, así como los requerimientos médicos mínimos para la realización del TCPH en algunos casos los tiempos de espera pueden prolongarse; ante esto, en caso de que el tiempo transcurrido entre la finalización del programa de entrenamiento y el ingreso a la UTMO fuera mayor a un mes, se reforzaron las técnicas cognitivo conductuales con el paciente a fin de evitar el desvanecimiento, para ello se programó una sesión previa a la hospitalización para reforzar la información de psicoeducación y se realizar ejercicios de relajación progresiva muscular pasiva. En caso contrario, que el tiempo entre la referencia para su evaluación y la fecha de hospitalización fuera menor a una semana, se ajustaron los tiempos entre las sesiones con el fin de brindar el programa completo pero en menor tiempo.

\section{EVALUACIÓN DE LOS RESULTADOS}

En la primera sesión se recabaron los datos sociodemográficos (edad, sexo, estado civil, religión, nivel de estudios, apoyo social y conciencia de enfermedad) y médicos (diagnóstico, tipo de trasplante, antecedentes psiquiátricos). De igual forma se llevó a cabo la aplicación de los instrumentos con los cuales se midieron las variables psicológicas:

- Para evaluar ansiedad y depresión, se utilizó la Escala Hospitalaria de Ansiedad y Depresión (HADS), en la versión adaptada a pacientes colombianos oncológicos que cuenta con un $\alpha=0,86^{(27)}$.

- Para medir estrés, se empleó el Termómetro de Distrés (DT) en su versión adaptada a pacientes mexicanos con cáncer, que tiene una sensibilidad de $93 \%$ y una especificidad de $76 \%{ }^{(28)}$.

Por otro lado se midió la necesidad de atención por parte del servicio de psiquiatría en un nivel dicotómico (Si/No); los criterios para solicitar la interconsulta fueron sí durante estancia hospitalaria el paciente presentaba en evaluación mediante el HADS puntuaciones mayores a ocho en ansiedad y nueve en depresión ${ }^{(27)}$ y cuatro o más en el $\mathrm{DT}^{(28)}$, o el médico tratante o personal de enfermería consideraban necesaria la valoración de psiquiatría ante el posible requerimiento de fármacos. En aquellos pacientes que fueron valorados por el servicio de psiquiatría durante la hospitalización se contabilizó el número de interconsultas recibidas, el diagnóstico emitido por dicho servicio y la medicación indicada. Cabe resaltar que con el objetivo de evitar sesgos en las evaluaciones, no se dio a conocer la realización de este estudio al servicio de psiquiatría.

\section{Características de los participantes}

Se evaluó a 23 pacientes candidatos a trasplante hematopoyético de células madre, de los cuales 21 fueron considerados aptos para ser sometidos a trasplante, de ellos 14 completaron el programa de intervención propuesto. En la Figura 1 se muestra las razones de muerte experimental de los sujetos iniciales. 
Figura 1. Causas de muerte experimental de los participantes a lo largo del estudio.

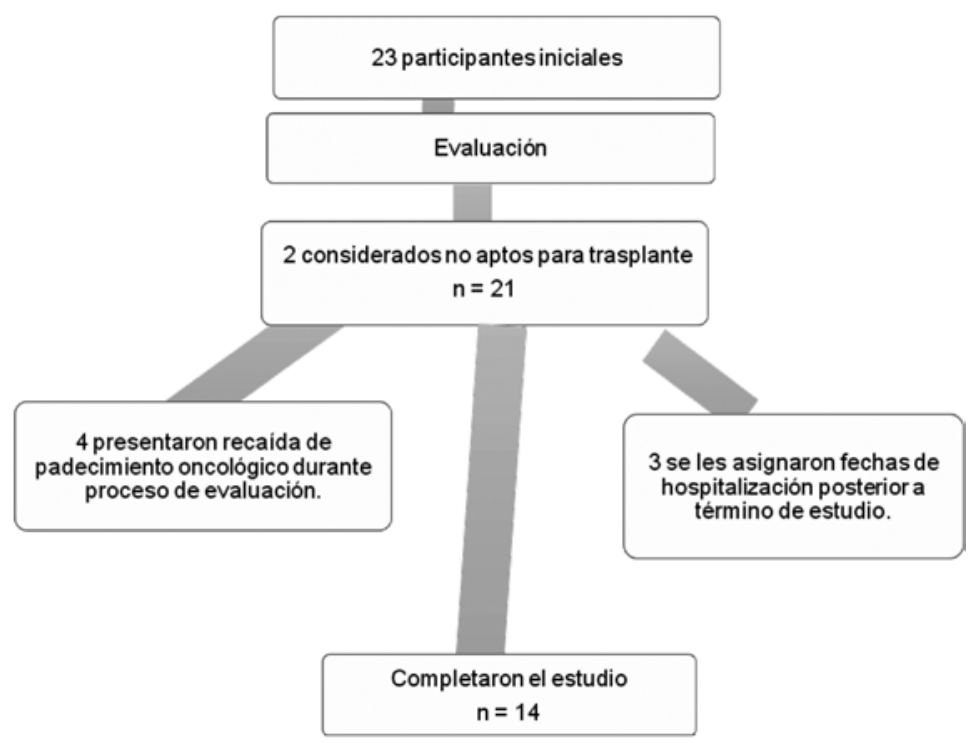

Por otro lado, el grupo histórico se conformó por 21 sujetos, cuyos características médicas y sociodemográficas se muestran en la tabla 2 junto al del grupo de intervención que completó el programa. Se puede observar que la media de edad del total de los participantes fue de 40 años, con valores similares en ambos grupos (43 en grupo de intervención y 39 en grupo histórico); el 97\% de los participantes tenían un diagnóstico oncológico, mientras que sólo una persona (3\%) del grupo control presentó anemia aplásica, que es un diagnóstico benigno en el que se indica el TCPH.

\section{RESULTADOS}

De los 14 pacientes que completaron la intervención, se encontró que cuatro (28,57\%) tenían antecedentes psicológicos patológicos previos a diagnóstico médico, de acuerdo con la información brindada por ellos, los diagnósticos establecidos fueron: (a) estrés en dos participantes, (b) trastorno por déficit de atención con hiperactividad en un caso y (c) trastorno límite de la personalidad en otro paciente. Por otro lado, dos $(14,28 \%)$ de los participantes tenían antecedentes familiares de tíos de línea materna con algún trastorno mental; uno de trastorno de depresión mayor y uno de trastorno de ansiedad sin especificar.

Al momento de la evaluación nueve $(64,28 \%)$ de los participantes contaban con noción de enfermedad; al ser capaces de describir de manera general el procedimiento médico a realizar, conocían de manera superficial los objetivos del trasplante y mencionaron parte de los efectos secundarios, principalmente aquellos relacionados con el uso de la quimioterapia. Se consideró que cuatro $(28,57 \%)$ tenían conciencia de enfermedad al identificar de manera puntual el objetivo del tratamiento y las probabilidades de éxito de acuerdo con su condición médica; describieron el procedimiento a realizar considerando tiempos, los riesgos generales del uso de quimioterapia y aquellos particulares en 


\section{Tabla 2. Características médicas y sociodemográficas de los participantes}

\begin{tabular}{|c|c|c|c|}
\hline & $\begin{array}{c}\text { Total } \\
(\mathrm{n}=35)\end{array}$ & $\begin{array}{c}\text { Grupo de } \\
\text { intervención } \\
(n=14)\end{array}$ & $\begin{array}{c}\text { Grupo control } \\
\text { histórico } \\
(n=21)\end{array}$ \\
\hline \multirow[t]{2}{*}{ Edad } & media (s.d.) & media (s.d.) & media (s.d.) \\
\hline & $40,9(14,2)$ & $43,6(15,45)$ & $39,05(13,46)$ \\
\hline Sexo & $f(\%)$ & $f(\%)$ & $f(\%)$ \\
\hline Mujer & $20(57,1)$ & $7(50)$ & $13(61,9)$ \\
\hline Hombre & $15(42,9)$ & $7(50)$ & $8(38,1)$ \\
\hline Estado civil & $f(\%)$ & $f(\%)$ & $f(\%)$ \\
\hline Soltero & $8(22,9)$ & $5(35,7)$ & $3(14,3)$ \\
\hline Casado & $17(48,6)$ & $6(42,9)$ & $11(52,4)$ \\
\hline Unión libre & $4(11,4)$ & $1(7,1)$ & $3(14,3)$ \\
\hline Separado & $6(17,1)$ & $2(14,3)$ & $4(19)$ \\
\hline Religión & $f(\%)$ & $f(\%)$ & $f(\%)$ \\
\hline Católico & $6(17,1)$ & $4(28,5)$ & $2(9,5)$ \\
\hline Cristiano & $27(77,1)$ & $8(57,1)$ & $19(90,5)$ \\
\hline Agnóstico & $2(5,7)$ & $2(14,3)$ & $0(0 \%)$ \\
\hline Nivel de estudios & $f(\%)$ & $f(\%)$ & $f(\%)$ \\
\hline Primaria & $7(20)$ & $2(14,3)$ & $5(23,8)$ \\
\hline Secundaria & $6(17,1)$ & $3(21,4)$ & $3(14,3)$ \\
\hline Técnico & $9(25,7)$ & $2(14,3)$ & $7(33,3)$ \\
\hline Preparatoria & $8(22,9)$ & $4(28,5)$ & $4(19)$ \\
\hline Licenciatura & $5(14,3)$ & $3(21,4)$ & $2(95,5)$ \\
\hline Diagnóstico & $f(\%)$ & $f(\%)$ & $f(\%)$ \\
\hline Linfoma de Hodgkin & $4(11,4)$ & $2(14,3)$ & $2(9,5)$ \\
\hline Leucemia & $9(25,7)$ & $2(14,3)$ & $7(33,3)$ \\
\hline Linfoma No Hodgkin & $11(31,4)$ & $6(42,9)$ & $5(23,8)$ \\
\hline Mieloma Múltiple & $10(28,6)$ & $4(28,5)$ & $6(28,6)$ \\
\hline Anemia aplásica & $1(2,9)$ & $0(0)$ & $1(4,8)$ \\
\hline \multicolumn{4}{|l|}{ Tipo de trasplante } \\
\hline Autólogo & $25(71,4)$ & $12(85,7)$ & $13(61,9)$ \\
\hline Alogénico & $10(28,6)$ & $2(14,3)$ & $8(38,1)$ \\
\hline \multicolumn{4}{|l|}{ Diagnóstico de psiquiatría } \\
\hline Sin integrar diagnóstico & $24(68,6)$ & $10(71,4)$ & $14(66,6)$ \\
\hline Reacción de ajuste a tratamiento & $1(2,9)$ & $0(0)$ & $1(4,8)$ \\
\hline Tr. depresivo recurrente & $1(2,9)$ & $0(0)$ & $1(4,8)$ \\
\hline Tr. de ansiedad generalizada & $2(5,7)$ & $1(7,1)$ & $1(4,8)$ \\
\hline Tr. afectivo & $1(2,9)$ & $0(0)$ & $1(4,8)$ \\
\hline Episodio depresivo leve & $1(2,9)$ & $0(0)$ & $1(4,8)$ \\
\hline Sintomatología ansiosa & $1(2,9)$ & $0(0)$ & $1(4,8)$ \\
\hline Estresores económicos & $1(2,9)$ & $0(0)$ & $1(4,8)$ \\
\hline Sintomatología depresiva & $1(2,9)$ & $1(7,1)$ & $0(0)$ \\
\hline Sintomatología afectiva & $1(2,9)$ & $1(7,1)$ & $0(0)$ \\
\hline Datos clínicos inespecíficos & $1(2,9)$ & $1(7,1)$ & $0(0)$ \\
\hline \multicolumn{4}{|l|}{ Medicamento psiquiátrico al ingreso } \\
\hline Sin tratamiento & $29(82,8)$ & $12(80,9)$ & $17(85,7)$ \\
\hline Sertralina & $3(8,6)$ & $0(0)$ & $3(14,3)$ \\
\hline Citalopram & $1(2,9)$ & $0(0)$ & $1(4,8)$ \\
\hline Mirtazapina & $1(2,9)$ & $1(2,9)$ & $0(0)$ \\
\hline Escitalopram + Alprazolam & $1(2,9)$ & $1(2,9)$ & $0(0)$ \\
\hline
\end{tabular}


relación al tipo de trasplante; así como los cuidados requeridos y los profesionales de salud a quien podrían acudir en caso de ser necesario.

En promedio, los participantes refirieron contar con una red de apoyo de 7,25 ( \pm $5,7)$ miembros; al evaluar la percepción de apoyo, se obtuvo una media de $8( \pm 0,6)$; cabe resaltar que el principal apoyo recibido fue emocional y el percibido como más difícil de obtener fue el instrumental, incluso en dos $(14,28 \%)$ de los casos dicho apoyo recaía en una sola persona.

En la tabla 3 se muestran los resultados obtenidos en la evaluación inicial; se puede observar que los participantes reportaron una media de 3,3 en valoración de distrés y un promedio de 6,6 problemas, siendo en orden de frecuencia los más importantes: (a) seguridad financiera; (b) miedo, principalmente a la necesidad de someterse a un tratamiento nuevo; (c) problemas de transporte; (d) problemas de memoria y concentración y (e) preocupaciones de diversas índoles. Por otro lado, en la escala de depresión del HADS se obtuvo una media de tres puntos y en la escala de ansiedad se muestra un promedio de cinco.

Con 10 participantes $(71 \%)$ se terminó el programa de intervención en los tiempos programados; en uno $(7,14 \%)$ se redujo el número de sesiones debido a la cercanía de la hospitalización y en tres $(21,4 \%)$ se reforzaron las técnicas planeadas ya que transcurrió más de un mes entre el término del programa de intervención y la fecha de ingreso a la unidad de trasplante. Durante el periodo de hospitalización, los pacientes realizaron el programa de actividad física 14 días en promedio, lo que representa una adherencia de $84 \%$ y no se presentaron complicaciones físicas derivadas de la actividad como dolor o sangrados.

En la figura 2 se muestran los promedios de las mediciones diarias de estrés (DT) durante la estancia hospitalaria; se puede observar un incremento en los días periféricos a la infusión de células madre (Día 0) y entre los días ocho y 11 , en los que se estima la recuperación hematológica. Los pacientes refirieron esos días como decisivos sobre la eficacia de trasplante refiriendo pensamientos como "ya quiero que peguen las células, así me empezaré a recuperar", "si injertó quiere decir que las células están funcionando".

Por otro lado, como se observa en la figura 3, se detectaron diferencias entre las mediciones de ansiedad y depresión mediante el HADS, con un incremento

\section{Tabla 3. Resultados de instrumentos de medición}

\begin{tabular}{lccc}
\hline \multicolumn{1}{c}{ Instrumento } & $\begin{array}{c}\text { Valores } \\
\text { Mínimos - Máximos }\end{array}$ & $\begin{array}{c}\text { Valores mínimos y } \\
\text { máximos del grupo }\end{array}$ & $\begin{array}{c}\text { Media aritmética } \\
\text { del grupo }\end{array}$ \\
\hline $\begin{array}{l}\text { Termómetro de estrés } \\
\text { Puntaje reportado }\end{array}$ & $0-10$ & $0-10$ & 3,3 \\
Total de problemas & $0-35$ & $0-16$ & 6,6 \\
$\quad$ & & 1,1 \\
$\quad$ Problemas prácticos & $0-5$ & $0-3$ & 0,18 \\
$\quad$ Problemas familiares & $0-2$ & $0-1$ & 2 \\
$\quad$ Problemas emocionales & $0-3$ & $0-5$ & 3,5 \\
$\quad$ Problemas físicos & $0-20$ & $0-9$ & \\
\hline Escala Hospitalaria de Ansiedad & & & 5 \\
y Depresión (HADS) & & & 3 \\
Ansiedad & $0-21$ & $0-15$ & \\
Depresión & $0-21$ & $0-11$ & \\
\hline
\end{tabular}


Figura 2. Promedio de mediciones diarias de distrés durante estancia hospitalaria del grupo de intervención $(n=14)$ (DT= Termómetro de distress)

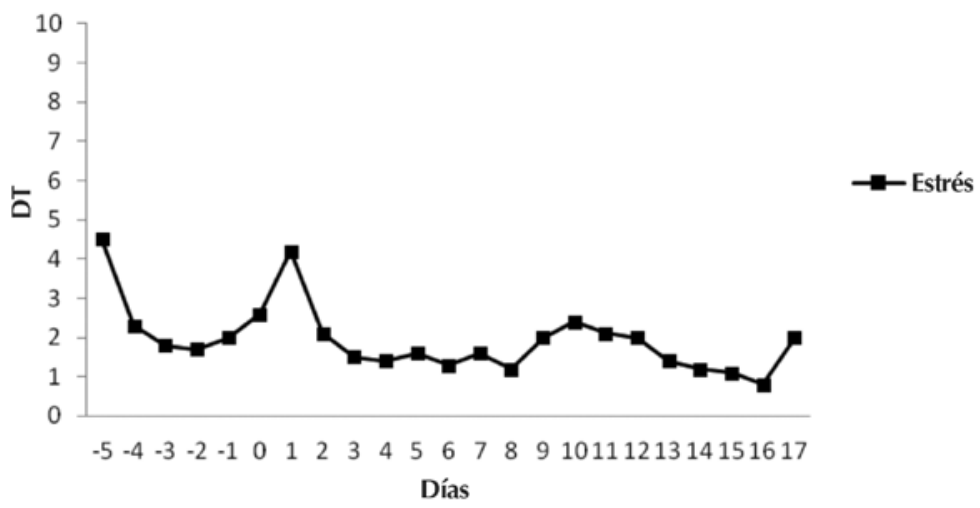

Figura 3. Mediciones con HADS en el grupo de intervención ( $n=14)$ a lo largo del estudio.

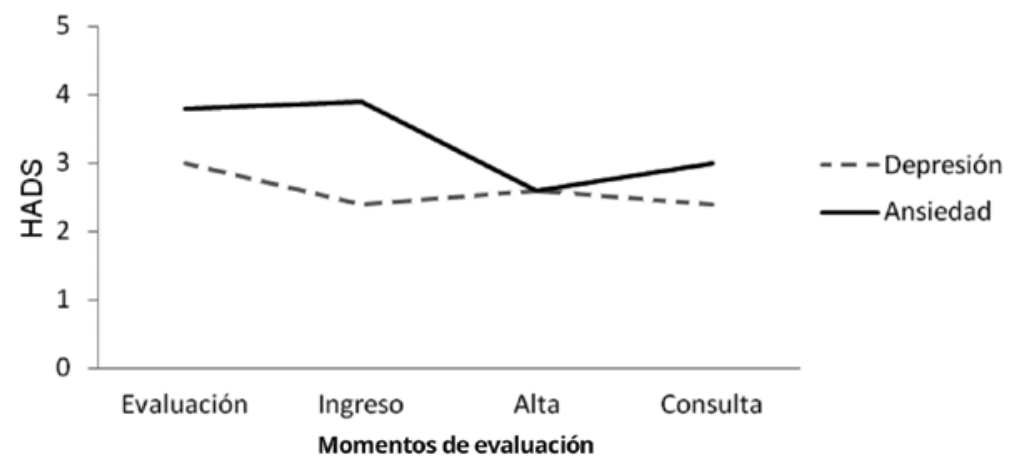

de ansiedad al momento del ingreso, la cual disminuye al alta y vuelve a tener un aumento en la consulta de seguimiento; mientras que la depresión tuvo un decremento al ingreso a la unidad de trasplante y mostró un incremento al alta.

Como parte de las comparaciones que se pudieron realizar entre el grupo de intervención y el grupo control histórico; en la figura 4 se observa que en el grupo histórico $12(57,1 \%)$ de los pacientes requirieron de interconsulta a psiquiatría durante la estancia hospitalaria; mientras que del grupo de intervención se solicitó el apoyo de este servicio en cinco casos
$(35,7 \%)$. Del total de pacientes que utilizaron los servicios de psiquiatría, el número de visitas de este servicio fue menor en el grupo de intervención con un promedio de 1,7 consultas por paciente, mientras que en el grupo histórico, fueron necesarias un promedio de tres visitas por sujeto (ver la figura 5).

Como resultado de las interconsultas de psiquiatría, se observó que en el grupo de histórico hubo nueve sujetos con sintomatología psicológica que no se explicaba por efectos secundarios del tratamiento médico y en tres sujetos no se integró diagnóstico psiquiátrico; mientras que en grupo de in- 
Figura 4. Porcentaje de pacientes que requirieron de interconsulta a psiquiatría durante hospitalización.

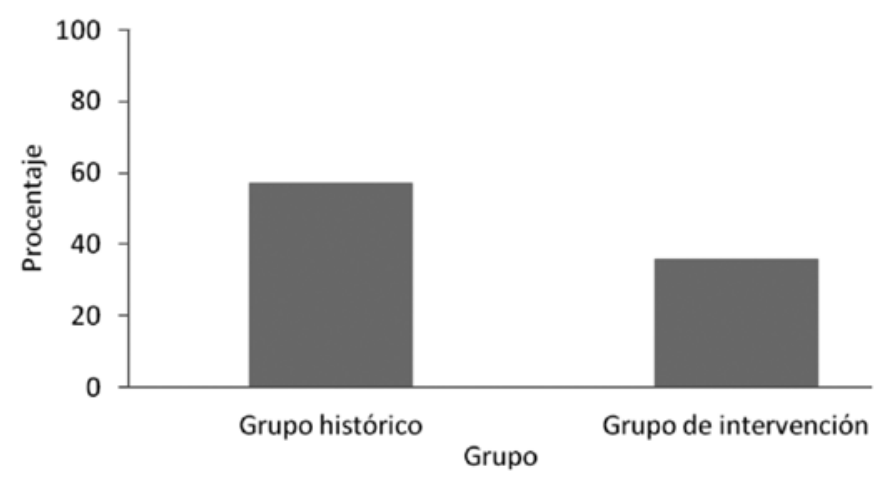

Figura 5. Promedio de consultas de psiquiatría por paciente durante hospitalización.

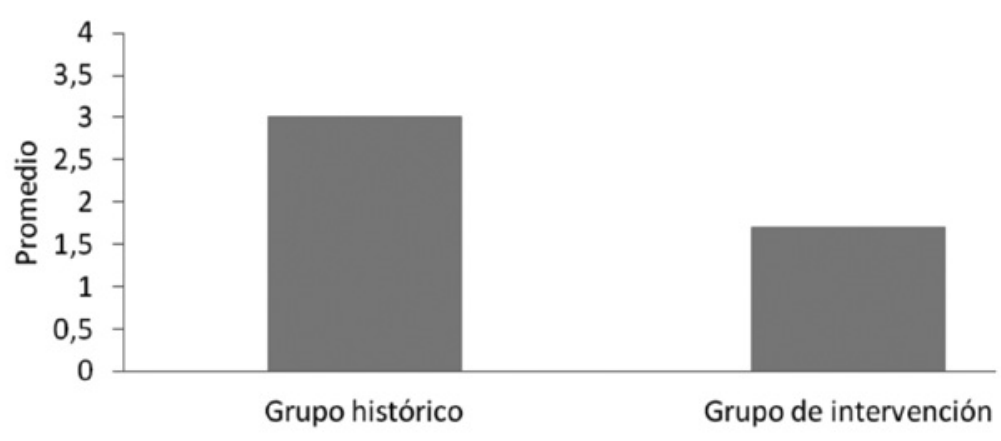

Grupos

tervención dos sujetos presentaron insomnio, de los cuales en un caso se consideró secundario a condición médica debido a reacción cutánea y en el otro sujeto se evaluó como no dependiente de condición médica relacionado con mala higiene de sueño, los otros dos sujetos fueron evaluados como estables. Con base en estos diagnósticos, el servicio de psiquiatría determinó el manejo psicofarmacológico de cada participante. En el grupo histórico, 17 pacientes ingresaron a la unidad de trasplante sin medicamentos, de éstos, cuatro $(23,5 \%)$ iniciaron tratamiento farmacológico; mientras que en el grupo de intervención de 12 participantes que ingresaron sin manejo psiquiátrico, en uno $(8,3 \%)$ se requirió el uso de mirtazapina como inductor de sueño con una dosis de $30 \mathrm{mg}$. (ver la figura 6).

Por otro lado, de los pacientes que ingresaron con medicamento psiquiátrico a la unidad de trasplante; en el grupo histórico, de cuatro sujetos, fue necesario incrementar la dosis en dos casos $(50 \%)$ y modificar el fármaco en uno (25\%); mientras que en el grupo de intervención de dos participantes que ingresaron con manejo farmacológico, se cambió el medicamento en uno $(50 \%)$ y en uno $(50 \%)$ no se presentó ninguna modificación (tabla 4).

Se consideraron los días de estancia hospitalaria por su estrecha relación con 
Figura 6. Porcentaje de pacientes que ingresaron sin tratamiento psicofarmacológico a la unidad de trasplante y fue necesario iniciar medicación durante estancia hospitalaria.

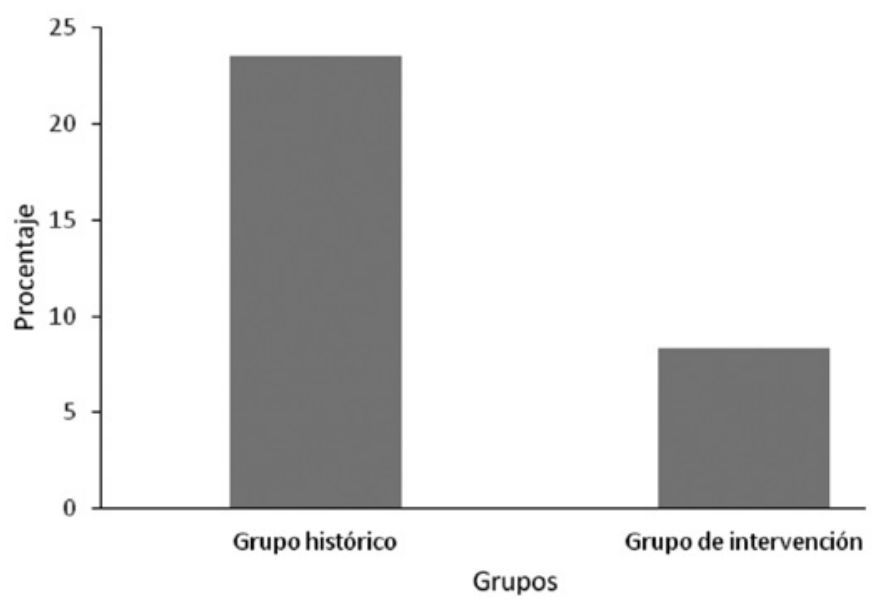

Tabla 4. Ajuste de medicamento psiquiátrico durante estancia hospitalaria

\begin{tabular}{llllllll}
\hline & \multicolumn{3}{c}{ Grupo histórico } & \multicolumn{3}{c}{ Grupo de intervención } \\
\hline \multicolumn{2}{c}{ Ingreso } & \multicolumn{2}{c}{ Alta } & \multicolumn{2}{c}{ Ingreso } & \multicolumn{2}{c}{ Alta } \\
\hline Medicamento & Dosis & Medicamento & Dosis & Medicamento & Dosis & Medicamento & Dosis \\
\hline Sertralina & $50 \mathrm{mg}$ & Sertralina & $125 \mathrm{mg}$ & Mirtazapina & $30 \mathrm{mg}$ & Mirtazapina & $30 \mathrm{mg}$ \\
Sertralina & $50 \mathrm{mg}$ & Clonazepam & $1,2 \mathrm{mg}$ & Escialopram + & $10 \mathrm{mg}$ & Mirtazapina & $30 \mathrm{mg}$ \\
Sertralina & $50 \mathrm{mg}$ & Sertralina & $50 \mathrm{mg}$ & Aloprazolam & & & \\
Citalopram & $20 \mathrm{mg}$ & Citalopram & $30 \mathrm{mg}$ & & & & \\
\hline
\end{tabular}

Figura 7. Días de estancia hospitalaria en grupos histórico y de intervención

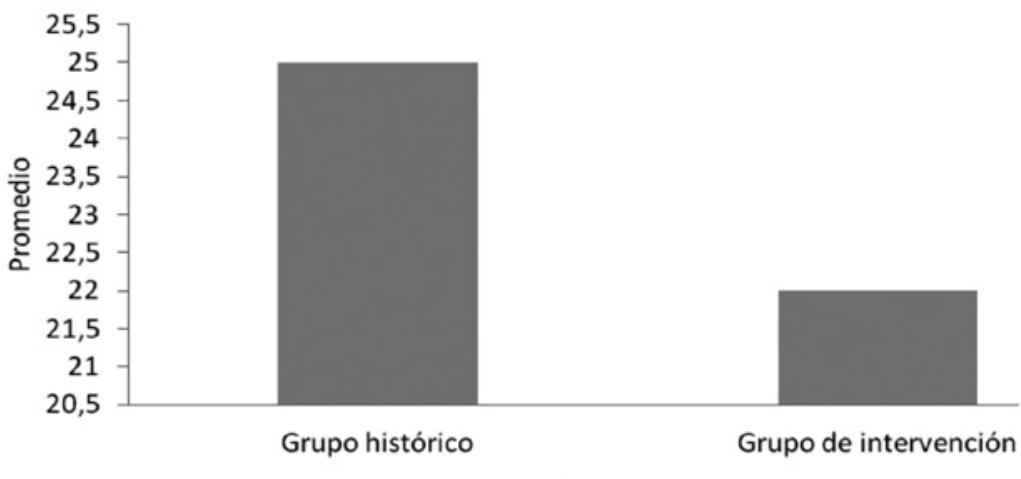


las complicaciones médicas por infección derivadas de la falta de adherencia a tratamiento, específicamente en cuidados de higiene. En la Figura 7 se muestra una diferencia de cinco días entre ambos grupos; el grupo histórico tuvo un promedio de 27,4 días y el grupo de intervención una media de 22 días.

\section{DISCUSIÓN}

El objetivo central de este estudio fue evaluar el efecto de una intervención cognitivo conductual y actividad física en variables psicológicas (ansiedad, estrés y depresión), así como variables médicas (estancia hospitalaria y comorbilidad psiquiátrica) durante el proceso de trasplante de médula ósea. De acuerdo con los datos obtenidos se pudo constatar que la intervención fue efectiva en mantener, de manera general, la estabilidad psicológica de los sujetos, a pesar de las variaciones observadas, al someterse a un tratamiento que se ha asociado a sintomatología psicosocial $^{(2,5)}$. Dichos resultados son apoyados por los efectos obtenidos en otros estudios en muestras e intervenciones similares ${ }^{(23,24)}$.

En el caso de ansiedad se observa un incremento al ingreso a la unidad, posteriormente disminuye al alta y se presenta un aumento en la consulta; mientras que las evaluaciones de depresión, se mantienen estables con un ligero descenso al ingreso. Dichos incrementos en ansiedad y estrés en los días periféricos a la infusión de células pueden ser explicados por la interrelación de múltiples factores: (a) malestares físicos como náusea, vómito y mareo derivados de los medicamentos quimioterapéuticos administrados; (b) disminución en la frecuencia y duración de las visitas del personal de enfermería y familiares por riesgo de infección; (c) incertidumbre sobre el procedimiento de infusión referido por los pacientes ${ }^{(29)}$ y en el caso particular de los participantes, se observó que las limitaciones de movilidad producidas a la baja en plaquetas, es referida como "de las peores cosas" ya que los obliga a permanecer en la habitación y en algunos casos realizar sus necesidades fisiológicas en productos de higiene.

La eficacia del programa puede ser explicada gracias a la incorporación de técnicas con diversos objetivos que favorecen la adaptación del sujeto a las condiciones del trasplante; de manera específica la psicoeducación adaptada al procedimiento médico favorece la adherencia a tratamiento e incrementan las habilidades de afrontamiento ${ }^{(30,31)}$ que se atribuye a darle a conocer con anticipación las condiciones requeridas, formular y resolver sus dudas con el personal médico y generar un plan de acción que abraque todo el proceso de trasplante.

La relajación progresiva muscular ha demostrado ser eficaz para reducir el consumo de oxígeno, ritmo cardiaco, tensión muscular y para normalizar la presión sanguínea durante la administración de quimioterapéuticos; así como para disminuir la intensidad y duración de los efectos secundarios como pérdida de apetito, pérdida de energía, náusea, reflujo y mucositits $^{(32)}$. Dichos síntomas suelen presentarse durante un trasplante de médula ósea, por lo que se puede inferir que al reducir la sintomatología antes mencionada, el paciente presenta mayor energía y menor malestar, lo que favorece la adherencia al programa de actividad física, que a su vez permite reducir la pérdida de fuerza muscular durante la hospitalización ${ }^{(24)}$. Se infiere que en conjunto las técnicas contribuyen a disminuir los efectos secundarios del tratamiento y favorecer la adaptación del sujeto.

Las interconsultas realizadas por psiquiatría fueron menores en el grupo de intervención; del mismo modo, los diagnósticos establecidos requirieron de menor incidencia de manejo psicofarmacológico, 
lo que disminuye las comorbilidades y complicaciones en seguimiento y por otro lado reduce los costos del procedimiento. Esto puede ser atribuido a la estabilidad emocional de los pacientes durante el procedimiento favorecida por el aprendizaje de las técnicas cognitivo conductuales y a que la mayoría de los procedimientos se Ilevaron a cabo sin complicaciones médicas. Hasta donde se conoce es el primer estudio que compara estas variables.

En el caso de los días de estancia hospitalaria, se encontró que fue menor por cinco días en el grupo de intervención, lo que pudiera estar relacionado con menor incidencia de infecciones asociadas a tratamiento médico y patógenos conductuales, como mucositis (asociada a pobre higiene bucal), infecciones urinarias (relacionada con baja ingesta de líquidos) y lesiones en piel (que pueden agravarse por rascarse o no hidratar adecuadamente la zona afectada ${ }^{(2)}$; en este sentido el uso de la relajación se ha asociado a un bajo número de infecciones en pacientes sometidos a trasplante de médula ósea ${ }^{(33)}$. Sin embargo, es necesario realizar estudios aleatorizados que generen evidencia en este sentido.

La selección del diseño se basó en consideraciones éticas, ya que no es posible agregar un grupo control debido a que actualmente no se cuenta con un protocolo de evaluación y el seguimiento durante la hospitalización se lleva a cabo en función de los requerimientos del personal de salud a cargo del paciente. Por otro lado, el uso de grupo en lista de espera es inviable ya que en la mayoría de los pacientes es un procedimiento al cual se someterán una sola vez, por lo que no se verían beneficiados en ningún momento con la intervención propuesta.

De igual forma, la población es limitada para la realización de diversas estrategias de intervención a comparar; por lo que se propuso este programa como un primer acercamiento; cuyos resultados podrían servir para el desarrollo de un protocolo durante un periodo más prolongado que permita la implementación de un diseño experimental capaz de generar resultados más confiables.

A pesar de las limitaciones, el presente estudio muestra congruencia con investigaciones previas ${ }^{(17-24)}$ lo que parece indicar una tendencia en la eficacia de programas cognitivos conductuales y la actividad física en la atención de pacientes sometidos a $\mathrm{TCPH}$, con predominio de programas multicomponentes ${ }^{(23,24)}$.

\section{Agradecimientos}

Informe de investigación derivado de proyecto de tesis para titulación de Programa de Maestría y Doctorado de Psicología del primer autor bajo la supervisión del último. Universidad Nacional Autónoma de México.

Se llevó a cabo con apoyo financiero del Consejo Nacional de Ciencia y Tecnología (CONACYT).

\section{REFERENCIAS BIBLIOGRÁFICAS}

1. American Cancer Society. Trasplante de células madre (trasplantes de sangre periférica, médula ósea y sangre del cordón umbilical). USA: American Cancer Society; 2012.

2. Apperley E, Carreras E, Gluckman E, Gratwoh A, Masszi T, editors. The EBMT Handbook. Haematopoietic Stem Cell Transplantation. $5^{\text {th }}$ ed. Evreux: European School of Haematology \& Eurupean Group for Blood and Marrow Transplantation; 2008.

3. Center for International Blood and Marrow Transplant Research. Current uses and outcome of hematopoietic stem cell transplantation 2011. USA: Center for International Blood and Marrow Transplant Research; 2011. 
4. Instituto Nacional de Cancerología. Informe de productividad de la Unidad de Trasplante de Médula Ósea 2012. Ciudad de México: Instituto Nacional de Cancerología; 2012.

5. Fritzsche K, Struss B, Stein B, Spahn C. Psychosomatic liaison service in hematological oncology: Need for psychotherapeutic interventions and their realization. Hematol Oncol 2003;21:83-9.

Doi:10.1002/hon.711

6. Syrjala KL, Langer SL, Abrams JR, Storer BE, Martin PJ. Late effects of hematopoietic cell transplantation among 10-years adult survivors compared with case-matched controls. J Clin Oncol 2005;23:6596606. Doi:10.1200/jco.2005.12.674

7. Savani BN, Montero A, Srinivasan A, Singh A, Shenoy A, Mielke S. et al. Chronic GVHD and pretransplantation abnormalities in pulmonary function are the main determinants predicting worsening pulmonary function in long-term survivors after stem cell transplantation. Biol Blood Marrow Transplant 2006;12:1261-9. Doi:10.1016/j.bbmt.2006.07.016

8. Bellver A, Moreno P. Riesgos psicosociales e intervención psicológica en los pacientes tratados de médula ósea. Psicooncología 2009;6:65-81.

9. Trask PC, Paterson A, Riva M, Brines B, Griffith K, Parker P, et al. Assessment of psychological distress in prospective bone marrow transplant patients. Bone Marrow Transplant 2002;29:917-25. Doi:10.1038/ sj.bmt. 1703557

10. Smith JM, McDonald RA. Renal transplantation in adolescents. Adolesc Med Clin 2005;16:201-4. Doi:10.1016/j. admecli.2004.09.003

11. Braamse AM, Gerrits MM, van Meijel B, Visser O, van Oppen P, Boenink AD, et al. Predictors of health-related quality of life in patients treated with auto- and allo SCT for hematological malignancies. Bone Marrow Transplant 2012;47:757-69. Doi:10.1038/bmt.2011.130
12. Schoulte JC, Lohnberg JA, Tallman B, Altmaier EM. Influence of coping style on symptom interference among adults recipients of hematopoietic stem cell transplantation. Oncol Nurs Forum 2011;38:582-6. Doi: 10.1188/11. ONF.582-586.

13. Jacobsen PB, Sadler IJ, Booth-Jones M, Soety E, Weitzner MA, Fields KK. Predictors of posttraumatic stress disorder symptomatology following bone marrow transplantation for cancer. J Consult Clin Psychol 2002;70:235-40.

14. Andorsky DJ, Loberiza FR, Lee SJ. Pretransplantation physical and mental functioning is strongly associated with self-reported recovery from stem cell transplantation. Bone Marrow Transplant, 2006;37:889-95. Doi:10.1038/ sj.bmt. 1705347

15. Sherman AC, Simonton S, Latif U, Spohn R, Tricot G. Psychosocial adjustment and quality of life among multiple myeloma patients undergoing evaluation for autologous stem cell transplantation. Bone Marrow Transplant 2004;33:955-62. Doi:10.1038/sj.bmt.1704465

16. Galindo-Vázquez $\mathrm{O}$, Pérez-Barrientos $\mathrm{H}$, Alvarado-Aguilar S, Rojas-Castillo E, Álvarez-Avitia MA, Aguilar-Ponce JL. Efectos de la terapia cognitivo conductual en el paciente oncológico: una revisión. GAMO 2013;12:108-15

17. Krasuska ME, Dmoszy ska A, Daniluk J, Stanislawek A. Information needs of the patients undergoing bone marrow transplantation. Ann Univ Mariae Curie Sklodowska 2002;57:178-85

18. Luebbert K, Dahnme B, Hasenbring M. The effectiveness of relaxation training in reducing treatment-related symptoms and improving emotional adjustment in acute non-surgical cancer treatment: a meta-analytical review. Psychooncology 2001;10:490-502. Doi:10.1002/pon.537

19. Kim SD, Kim HS. Effects of relaxation breathing exercise on anxiety, depression, 
and leukocyte in hematopoietic stem cell transplantation patients. Cancer Nurs 2005; 28: 79-83.

20. Courneya KS, Keats MR, Tuner AR. Physical exercise and quality of life in cancer patients following high dose chemotherapy and autologous bone marrow transplantation. Psychooncology 2000;9:127-36. Doi:10.1002/(SICI)1099$1611(200003 / 04) 9: 2<127::$ AID PON438>3.0.CO;2-L

21. Wiskemann J, Dreger $P$, Schwerdtfeger R, Bondong A, Huber G, Kleindienst N, et al. Effects of a partly self-administered exercise program before, during, and after allogeneic stem cell transplantation. Blood 2011; 117:2604-13. Doi:10.1182/ blood-2010-09-306308

22. Bloomfield SA, Coyle EF. Reposo en cama, declive del entrenamiento y mantenimiento de las adaptaciones inducidas por el entrenamiento. En: American College of Sport Medicine. Manual de consulta para el control y prescripción de ejercicio. Barcelona: Paidotribo; p.127-40; 2000

23. Jarden M, Hovgaard D, Boesen E, Quist M, Adamsen L. Pilot study of a multimodal intervention: mixed-type exercise and psychoeducation in patients undergoing allogeneic stem cell transplantation. Bone Marrow Transplant 2007;40:793-800. Doi:10.1038/sj.bmt.1705807

24. Jarden M, Baadsgaard MT, Hovgaard Dj, Boesen E, Adamsen L. A randomized trial on the effect of a multimodal intervention on physical capacity, functional performance and quality of life in adult patients undergoing allogeneic SCT. Bone Marrow Transplant 2009;43:725-37. Doi: 10.1038/bmt.2009.27

25. Medina C, Barquera S, Janssen I. Encuesta Nacional de Salud y Nutrición 2012. Instituto Nacional de Salud Pública.
Ciudad de México: SSA; 2012. Informe General ENSANUT

26. Reynoso L. Manual ilustrado de actividad física. México: UNAM; 2012

27. Rico JL, Restrepo M, Molina M. Adaptación y validación de la escala hospitalaria de ansiedad y depresión (HADS) en una muestra de pacientes con cáncer del Instituto Nacional de Cancerología de Colombia. Avances Medición 2005;3:73-86

28. Almanza-Muñoz JJ, Juárez IR, Pérez S. Traducción, adaptación y validación del termómetro de distrés en una muestra de pacientes mexicanos con cáncer. Rev Sanid Milit Mex 2008;62:209-17

29. Fife BL, Huster GA, Cornetta KG, Kennedy VN, Akard LP, Broun ER. Longitudinal study of adaptation to stress of bone marrow transplantation. J Clin Oncol 2000;18:1539-49

30. Husson O, Mols F, van de Poll Franse LV. The relation between infromation provision and health-related quality of life, anxiety and depression among caner survivors: A systematic review. Ann Onc 2010;22:761-72. Doi:10.1093/annonc/ mdq413

31. O'Connor G, Coates V, O'Neill S. Randomised controlled trial of a tailored information pack for patients undergoing surgery and treatment for rectal cancer. Eur J Oncol Nurs 2014; 18: 183-91. Doi:10.1016/j.ejon.2013.10.011

32. Song $\mathrm{QH}, \mathrm{Xu} \mathrm{RM}$, Zhang $\mathrm{QH}, \mathrm{Ma} \mathrm{M}$, Zhao XP. Relaxation training during chemotherapy for breast cancer improves mental health and lessens adverse events. Int J Clin Exp Med 2013;6:979-84

33. Pulgar A, Garrido S, Alcalá A, Reyes del Paso GA. Psychosocial predictors of immune response following bone marrow transplantation. Behav Med 2012;38:12-8. Doi: 10.1080/08964289.2011.647118 
